\title{
Maltrato infantil y representaciones de apego: defensas, memoria y estrategias, una revisión*
}

Child Maltreatment and Attachment Representations:
Defenses, Memory and Strategies, a Review

Recibido: diciembre 15 de 2010 | Revisado: julio 8 de 2011 | Aceptado: enero 30 de 2012

\author{
ANDRÉS FRESNO RODRÍGUEZ ** \\ ROSARIO SPENCER CONTRERAS *** \\ TANia Retamal CASTRO ***** \\ Universidad de Talca, Chile
}

SICI: 1657-9267(201209)11:3<829:MIYRDA >2.0.TX;2-J

Para citar este artículo. Fresno, A., Spencer, R. \& Retamal, T. (2012). Maltrato infantil y representaciones de apego: defensas, memoria y estrategias, una revisión. Universitas Psychologica, 11 (3), 829838.

* Artículo de revisión teórica. El presente artículo forma parte de las actividades del proyecto de investigación en curso (noviembre de 2008-noviembre de 2011) № 11080107 "Apego y maltrato infantil: Estudio de la influencia de distintos tipos de maltrato sobre la calidad de las representaciones de apego infantil" financiado por el Fondo Nacional de Desarrollo Científico y Tecnológico del Gobierno de Chile (FONDECYT), que cuenta con el patrocinio de la Universidad de Talca y del Servicio Nacional de Menores del Gobierno de Chile (SENAME), y la colaboración de la Fundación CRATE. Este artículo contó con el apoyo del Programa de Investigación en Calidad de Vida y Ambientes Saludables de la Facultad de Psicología de la Universidad de Talca.

** Facultad de Psicología. Tel.: (056-9) 78065738. Email: andresfresno@gmail.com

**** Facultad de Psicología. Tel.: (056-9) 98293957. Email: rosariospencer@gmail.com

***** Facultad de Psicología. Tel.: (056-9) 89566522. Email: taniaretamal@gmail.com

\section{RES UMEN}

El presente artículo realiza una revisión de la literatura sobre la influencia del maltrato infantil en la conformación de las representaciones del apego. Se describen los principales postulados teóricos respecto de los efectos del maltrato en los procesos defensivos, los sistemas de memoria y las estrategias de apego, elementos discutidos a través de la presentación de un modelo integrativo, el cual es contrastado con estudios empíricos. Se constata que el maltrato infantil afecta la calidad de las representaciones del apego, sin embargo se aprecia que los estudios no logran dar cuenta de toda la complejidad del fenómeno del maltrato. Los postulados teóricos abordados en este trabajo son marginalmente puestos a prueba en las investigaciones que se reportan.

Palabras clave autores

Representaciones de apego, procesos defensivos, memoria, estrategias de apego.

Palabras clave descriptores

Psicología del desarrollo, maltrato infantil, revisión.

\section{A B S T R A C T}

The aim of this paper is a review of the literature about the influence of child abuse on the formation of attachment representations. It describes the main theoretical assumptions about the effects of abuse on the defensive processes, memory systems and attachment strategies. These elements are discussed trough the presentation of an integrative model which is contrasted with empirical studies. It shows that child abuse affects the quality of attachment representations; however the studies do not take in to account all the complexity of the phenomenon of abuse. Theoretical issues discussed in this paper are marginally tested in the research reported.

Key words authors

Attachment representations, defensive processes, memory, attachment strategies.

Key words plus

Development psychology, child maltreatment, review. 
La literatura afirma que el maltrato afecta negativamente el desarrollo de los niños (Cicchetti \& Toth, 2005; Hildyard \& Wolfe, 2002; Howe, 2005; Macfie, Cicchetti \& Toth, 2001 Moreno Manso, 2005; Pollak, Cicchetti, Hornung \& Reed, 2000), y la calidad del apego infantil no es la excepción (Aspelmeier, Elliot \& Smith, 2007; Baer \& Martinez, 2006; Bowlby, 1969, 1973, 1980; Glaser, 2000; Hodges \& Steele, 2000; Morton \& Browne, 1998). Casi la totalidad de los niños víctimas de maltrato desarrollan un apego inseguro-desorganizado (Barnett, Ganiban \& Cicchetti, 1999; Carlson, 1998; Cicchetti \& Barnet, 1991; van IJzendoorn, Schuengel \& Bakermans-Kranenburg, 1999), lo que impacta negativamente en su desarrollo posterior (Cicchetti \& Toth, 2005; Hesse \& Main, 2000; Jacobvitz \& Hazen, 1999; Solomon \& George, 1999). No obstante, las investigaciones sobre la influencia del maltrato en el apego infantil se centran en su dimensión comportamental, existiendo escasa literatura sobre la relación entre maltrato y las representaciones del apego en niños (Baer $\&$ Martinez, 2006; Hodges \& Steele, 2000; Morton \& Browne, 1998; Toth, Cicchetti, Macfie, Maughan \& Vanmeenen, 2000).

Este artículo tiene como objetivo realizar un estado del arte acerca de los efectos del maltrato sobre las representaciones del apego analizando su impacto sobre las defensas, los sistemas de memoria y las estrategias de adaptación infantiles, para lo cual se revisa sucintamente el concepto de modelo interno operante de apego (Bowlby, 1969, 1973, 1980), luego se abordan aquellos elementos que, estando relacionados con las representaciones de apego, serían afectados por experiencias de maltrato, y finalmente se propone un modelo teórico integrativo que será discutido a partir de la teoría y los estudios empíricos.

\section{Las representaciones de apego: Modelos Internos Operantes}

Bowlby $(1969,1973,1980)$ plantea que los individuos desarrollan representaciones mentales o "Modelos Internos Operantes" (MIOs) de sí mismos, de su cuidador y de la relación entre ambos. Su construcción se realiza a partir de las características de las respuestas del cuidador a las necesidades de apego expresadas por el niño. El desarrollo del MIO de sí mismo y de la figura de apego es complementario, representando los dos extremos de un mismo vínculo (Bretherton \& Munholland, 2008).

Los MIOs comprenden aspectos afectivos y cognitivos que permiten evaluar las experiencias y comportamientos del individuo (Bowlby, 1969, 1980; Main, Kaplan \& Cassidy, 1985). Estos modelos permitirían predicciones y simulaciones de situaciones a nivel mental respecto de los comportamientos del cuidador y de los otros, como también las conductas, pensamientos y emociones del self (Bretherton, 1990; Bretherton \& Munholland, 2008). Cuando un niño cuenta con figuras de apego que responden a sus comportamientos de apego desarrollará representaciones del self, de los cuidadores y del entorno seguras, positivas y benignas (Bowlby, 1973, 1980; Bretherton, 1990, 2005; Bretherton \& Munholland, 2008; Main \& Hesse, 1990). Con el tiempo los MIOs se vuelven estables y resistentes a los cambios (Bowlby, 1969, 1980; Bretherton \& Munholland, 2008), sin embargo pueden modificarse producto de vivencias traumáticas como el maltrato (Bowlby, 1980; Weinfield, Sroufe \& Engeland, 2000).

\section{Representaciones de apego y maltrato}

\section{Procesos defensivos}

Bowlby (1980) plantea que en situaciones de maltrato tempranas los niños desarrollarían procesos defensivos para enfrentarlas, tales como la exclusión defensiva y la segregación del sistema principal (Bretherton \& Munholland, 2008).

La exclusión defensiva busca proteger al individuo de ser consciente de eventos o pensamientos que puedan ser insoportables si son aceptados como verdaderos (Bowlby, 1980). En el caso de niños maltratados, sobre todo si los padres no quieren hablar al respecto o cuando éstos niegan, minimizan o ridiculizan las necesidades de apego o los estados emocionales de sus hijos, los niños desarrollarían dos conjuntos de MIOs en conflicto (Bowlby, 1973, 
1980): el primero es excluido defensivamente de la conciencia y representa a un padre "malo", puesto que se basa en la experiencia adversa del niño con éste, y el segundo permanece consciente y asequible, y corresponde a la imagen de un padre "bueno", o representa lo que el padre quiere que el niño crea sobre él, caso en el cual se considera un solo self que es protegido a través de la exclusión de la representación parental negativa reemplazándola por una positiva idealizada (Bowlby, 1980; Bretherton \& Munholland, 2008).

La exclusión defensiva puede manifestarse a través de una desactivación del sistema de comportamientos de apego o por una desconexión cognitiva (Bowlby, 1980). En la desactivación los comportamientos, pensamientos y emociones relacionadas con el apego dejan de ser experimentadas, lo que resulta en una desconexión emocional, y en el caso de la desconexión cognitiva la persona separa la situación interpersonal ansiógena del conjunto de afectos y comportamientos que tal situación genera. Dichos elementos desconectados pueden ser interpretados de una forma menos amenazante, sin embargo esto implica una interpretación errónea de los mismos. Si la exclusión defensiva se ha dado de manera sistemática y persistente la desconexión cognitiva puede manifestarse a través de la interpretación equívoca de la situación que causa un afecto negativo, o dirigiendo hacia una persona cualquiera o hacia sí mismo los sentimientos negativos generados por el cuidador (Bowlby, 1980; Bretherton \& Munholland, 2008; Solomon \& George, 1999).

El segundo proceso defensivo corresponde a la segregación del sistema principal de representación del apego (Bowlby, 1980), que implica el desarrollo de múltiples selfs separados con acceso a diferentes MIOs organizados, y con un cúmulo de recuerdos propios. Dichos modelos pueden alternar su acceso a la consciencia: mientras uno domina la dirección de la conducta y los pensamientos, los otros permanecen desactivados total o parcialmente (Bretherton \& Munholland, 2008).

Complementando lo anterior, Crittenden (1990) señala que los procesos defensivos pueden ser concebidos como procesos que generan MIOs distorcionados, ya que cierta información relacionada con el apego es excluida de la percepción, o se crean recuerdos distorsionados de los cuidadores. Sin embargo esta autora considera que los casos descritos corresponderían al desarrollo de un $\mathrm{MIO}$ correcto que da cuenta de una relación distorsionada real. Por otra parte, Howe (2005) recalca que los procesos defensivos deben ser comprendidos de manera evolutiva dentro de la relación niño-cuidador. De esta forma, un cuidador con un apego preocupado responderá de manera inconsistente a las necesidades de apego de su hijo, quien hará frente a esta situación con una desconexión cognitiva, manifestada por una hiperactivación del sistema de apego con el fin de lograr el acercamiento con el cuidador. Si el cuidador posee apego evitativo, que no responde a las demandas de apego del niño, éste reaccionará desconectando su sistema de comportamientos de apego, emitiendo conductas evitativas en situaciones en que debiera activarse el sistema de apego. Finalmente, cuando el cuidador tiene un apego desorganizado el niño responde mediante la segregación del sistema representacional principal creando múltiples sistemas representacionales, segregados, e incoherentes entre sí, percibiéndose como conductas erráticas e incoherentes del menor (Howe, 2005 \& Liotti, 1999, 2004).

\section{Sistemas de memoria, estructuración de los MIOs y maltrato}

Tulving (1972) distingue la memoria episódica o autobiográfica, y la memoria semántica o ideas generalizadas sobre el mundo adquiridas por la experiencia o la relación con otras personas. Considerando esta distinción, Bowlby (1980) establece que la exclusión de los MIOs en conflicto responde a una codificación diferencial de una misma experiencia. Específicamente, los cuidadores que prohíben a los niños preguntarse por la ocurrencia de algún suceso negativo los obligan a excluir o separar dicho recuerdo almacenado en la memoria episódica, por lo tanto la representación que permanece disponible a la conciencia es una versión equivocada que los cuidadores le entregan al niño sobre lo sucedido, que se almacena a nivel de la memoria semántica, y que producida por el discurso entregado por los cuidadores se superpone al 
registro episódico perturbando la configuración de los MIOs. No obstante, aun cuando un $\mathrm{MIO}$ queda excluido, éste continua desarrollándose a lo largo de la vida e interviniendo en las conductas de los individuos (Bowlby, 1973).

Por su parte, Bretherton (1990, 2005) se basa en los estudios sobre la representación de eventos de Nelson (1986), y en el procesamiento de la memoria a través de esquemas de Shank (1982) para entender los MIOs y los procesos defensivos de los niños víctimas de maltrato planteados por Bowlby (1980). Respecto de los MIOs Bretherton (1990; Bretherton \& Munholland, 2008) propone que existe una jerarquía interconectada de esquemas o guiones que almacenan una secuencia de experiencias del niño. En concreto, en un nivel inferior se encuentran los esquemas interaccionales más cercanos a la experiencia con el cuidador (cuando me lastimo mi mamá siempre viene a ayudarme); luego, existe otro nivel que contiene un esquema general que engloba los niveles inferiores de experiencia con el cuidador (mi mamá siempre está disponible cuando la necesito), y finalmente, en un nivel superior, se encuentran esquemas más amplios que reúnen el contenido de los anteriores (mi mamá es una persona cariñosa). Así, lo que diferenciaría un $\mathrm{MIO}$ seguro de uno inseguro es su contenido, organización interna y consistencia relativa entre los niveles jerárquicos.

En relación a los procesos defensivos Bretherton (1990) señala que fragmentos de la memoria episódica están configurados en esquemas, y que éstos últimos están interconectados en jerarquías de información. El contenido que es excluido si bien no puede ser recuperado, se mantiene al interior de los esquemas, influyendo en la formación de éstos, influencia que hace que el modelo sea internamente contradictorio e incoherente. En concreto, se estima que los niños que desarrollan $\mathrm{MIOs}$ desorganizados tienen disociado algún esquema o la red de esquemas al interior de los niveles jerárquicos, siendo aquí donde la actualización de la información que se efectúa en un nivel puede que no se propague a los demás niveles. Por otro lado, cuando se trata de esquemas acerca de un cuidador idealizado puede que la información sea codificada como real, lo cual se manifiesta a través de alteraciones en la interpretación y en los comportamientos de apego emitidos. En los casos de quienes desarrollan un apego inseguro ambivalente evidencian una dificultad para generalizar la información desde los niveles de recuerdos autobiográficos para establecer esquemas generales abstractos, y en relación a los sujetos con apego inseguro evitativo, al parecer, mantienen divididos los esquemas dentro y a través de los niveles jerárquicos, por lo que la activación de un esquema no afectaría a otros (Bretherton, 1990).

\section{MIOs, estrategias de apego y maltrato}

Otra aproximación para comprender la conformación de los MIOs y su relación con el maltrato ha sido la de las "estrategias de apego" (Kobak, 1999; Kobak, Cole, Ferenz-Gillies, Fleming \& Gamble, 1993; Main, 1990), la cual señala que los patrones de interacción padre-hijo se construyen y autorregulan de manera particular y se vuelven una característica estable de la personalidad en contextos relacionales (Bowlby, 1973). Para que los patrones de interacción se autorregulen el niño debe desarrollar un $\mathrm{MIO}$ de las respuestas del cuidador y estrategias apropiadas para regular los comportamientos de apego (Kobak et al., 1993).

Cuando un niño percibe señales de amenaza y tiene una representación de un cuidador disponible pone en acción una serie de conductas para restablecer la cercanía con la figura de apego y ser calmado emocionalmente para luego poder volver a explorar, patrón de comportamiento denominado "estrategia primaria" (Main, 1990), que coincide con el patrón de comportamiento de apego seguro descrito por Ainsworth, Blehar, Waters y Wall (1978) en la situación extraña. Al contrario, cuando la figura de apego se comporta de manera insensible a las demandas de seguridad del niño este desarrolla patrones alternativos de comportamiento con el fin de hacer frente a la sensación de amenaza sin poner en riesgo la relación con la figura de apego (Main, 1990). Dichos patrones son llamados estrategias de apego secundarias y corresponden a la desactivación e hiperactivación del sistema de comportamientos de apego (Main, 
1990), cuya selección reside en el MIO desarrollado. En la estrategia de desactivación el niño opta por desactivar su sistema de apego para evitar conflictos con su cuidador y el dolor ante el rechazo de sus demandas (Howe, 2005), desarrollando un patrón de apego evitativo (Kobak et al., 1993). En el caso de la estrategia de hiperactivación el niño tiene una representación que las respuestas de la figura de apego son inconsistentes, caso en el cual sobre activa sus comportamientos de apego para lograr movilizar efectivamente las respuestas del cuidador (Howe, 2005), desarrollando un patrón inseguro ambivalente (Kobak et al., 1993). Tanto la estrategia primaria como la secundaria constituyen patrones organizados de respuesta a las situaciones de apego, son estables, perduran a lo largo del tiempo (Howe, 2005; Kobak et al., 1993; Main, 1990; Mikulincer \& Shaver, 2008) y buscan maximizar los cuidados parentales, adaptándose al estilo de cuidado parental (West \& George, 1999).

En el caso que las respuestas del cuidador sean extremadamente incoherentes y/o maltratadoras el niño no sería capaz de conformar una estrategia organizada de apego, situación ante la cual se activarían dos sistemas de comportamiento contradictorios entre sí: el de apego y el de miedo (Main \& Hesse, 1990), experimentando a la vez dos comportamientos incompatibles: el de escape y acercamiento (Lyons-Ruth, Bronfman \& Atwood, 1999; van IJzendoorn et al., 1999).

No obstante lo anterior, algunos autores proponen que los niños con apego desorganizado desarrollan frágiles estrategias de comportamiento para adaptarse a contextos de maltrato que les permiten, en cierta medida, protegerse y autorregularse (Crittenden, 1999; Liotti, 1999, 2004; West \& George, 1999).

\section{Discusión}

Para Bowlby $(1969,1973,1980)$ el objetivo central del apego es la supervivencia de la especie a través de la protección parental. En este sentido, los comportamientos de apego están organizados para asegurar la disponibilidad del cuidador cuando el niño se sienta en dificultades. La organización de los componentes del apego depende y está dirigida por las características de los MIOs basados en la interacción niño-cuidador.

Tomando en cuenta la teoría, para comprender la conformación y funcionamiento de los MIOs existen diversos niveles de análisis, como los considerados en esta revisión (defensas, sistemas de memoria y estrategias de apego), los cuales se ven influenciados negativamente por las experiencias de maltrato (Belsky \& Fearon, 2008; Bowlby, 1980; Bretherton \& Munholland, 2008; Crittenden, 1990; Howe, 2005; Liotti, 2004; Lyons-Ruth \& Spielman, 2004; Main \& Hesse, 1990). Si bien se señalan relaciones entre dichos niveles, hasta el momento no se cuenta con una propuesta teóricoempírica que los conjugue explícitamente en los casos de maltrato infantil (Bowlby, 1980; Bretherton \& Munholland, 2008; Crittenden, 1990; Liotti, 1999, 2004; Lyons-Ruth et al., 1999; Lyons-Ruth \& Spielman, 2004; Howe, 2005; Solomon \& George, 1999), para lo cual postulamos que la integración de los distintos niveles propuestos favorece la comprensión del proceso de conformación de los $\mathrm{MIOs}$ y de cómo éstos se ven afectados por dicho maltrato.

Así, el niño víctima de maltrato responde con la activación de un proceso defensivo particular para disminuir su sufrimiento, mantener la cercanía con su figura de apego y aumentar las probabilidades de supervivencia (Bowlby, 1969, 1973, 1980). El tipo de defensa puesto en marcha estará determinado por las características particulares de las respuestas del cuidador a las necesidades de apego del niño (Bowlby, 1980; Bretherton \& Munholland, 2008; Howe, 2005; Solomon \& George, 1999).

Frente a un rechazo de las necesidades se activará la desconexión del sistema de apego, dejando de experimentar conscientemente distrés en la activación del sistema y alivio en la desactivación de éste. En el caso de respuestas inconsistentes se responderá con una desconexión cognitiva, con lo cual se interrumpe la relación entre los afectos y comportamientos generados en una situación donde se activa el apego y la situación interpersonal que lo genera. Finalmente, en situaciones donde se evidencie una incoherencia extrema en las respuestas y/o se genere miedo en el niño cuando éste busca 
seguridad la reacción será la segregación del sistema principal del apego, registrando la información de la relación de apego en sistemas representacionales no integrados e incoherentes entre sí (Bowlby, 1980; Bretherton \& Munholland, 2008; Howe 2005). Los procesos defensivos modifican el registro de la información interna y externa alterando el procesamiento integrado de la información en los distintos sistemas de memoria (Bowlby, 1990), la cual queda organizada de manera parcial y segregada en distintos grados según sea la defensa, perturbando el registro de nueva información así como la evocación consciente de la misma (Bowlby, 1990; Bretherton, 1990; Bretherton \& Munholland, 2008). En consecuencia, las representaciones que conforman los MIOs en la memoria serán más o menos coherentes, integrados y con un mayor o menor acceso a la conciencia según sean los procesos defensivos activados. En el caso de la desconexión del sistema de comportamiento de apego y la cognitiva existiría la representación de un sólo self y de distintas representaciones de la figura de apego (Bowlby, 1980), lo que se podría traducir en que existiría una integración en los distintos sistemas de memoria respecto al sí mismo, pero no respecto a la figura de apego, alterando el registro y recuperación de información de ese polo del MIO. En el caso de la segregación del sistema principal la desconexión entre los distintos sistemas y niveles del sistema de memoria es total o casi total (Bretherton, 1990; Bretherton \& Munholland, 2008), postulando la existencia de múltiples selfs con sus correspondientes MIOs (Bowlby, 1980).

Una vez configurados los MIOs, estos dirigirán el sistema de comportamientos de apego configurando así una estrategia de apego particular (Bowlby 1980; Kobak, 1999; Kobak et al., 1993; Main, 1990). En el caso de los dos procesos concernientes a la exclusión defensiva y sus consecuentes procesamientos de la información, resultarán estrategias de apego secundarias (evitativo e hiperactivado) (Bowlby, 1980; Howe, 2005; Kobak et al., 1993; Main, 1990), y en el caso de la segregación del sistema principal de apego las representaciones del apego entregan información que activan de manera simultánea sistemas de comportamiento de apego contradictorios, originando un patrón de apego desorganizado (Main, 1990; Solomon \& George, 1999).

De este modo es posible postular que los niveles de análisis descritos se configuran de manera simultánea y complementaria a partir de las interacciones tempranas niño-cuidador, con el objetivo de responder adaptativamente a las relaciones interpersonales que se producen. Además, en la medida que aumenta la incoherencia de las respuestas del cuidador a las necesidades de apego del niño la calidad del apego se orienta hacia el polo de la inseguridad, en términos de defensas, procesamiento de la información y estrategias de apego.

Si bien lo anterior permite explicar una parte de los efectos del maltrato en el desarrollo de los MIOs, surgen interrogantes que evidencian la necesidad de mayor precisión del modelo. En primer lugar se hace necesario explicar a qué se refieren los estudios al hablar de maltrato, con el fin de examinar qué tipo de maltrato impacta el apego y cómo lo hace. La literatura señala que distintos tipos de maltrato así como sus configuraciones (duración, frecuencia, gravedad, vínculo con el perpetrador, etc.) tienen efectos particulares en el desarrollo psicológico (Ciccetti \& Toth, 2005; English et al., 2005; Lau et al., 2005; Litrownik et al., 2005; Manly, 2005). Si bien los planteamientos teóricos expuestos no permiten dar cuenta del modo en que se configuran los MIOs tomando en cuenta la complejidad del maltrato, Bowlby (1980) se aproxima al tema proponiendo que en casos de abuso sexual, donde los padres tienen un discurso que contradice o niega dicha experiencia del niño, se alteraría el registro en los distintos sistemas de memoria generando $\mathrm{MIOs}$ no integrados, y en términos de las defensas esta situación correspondería a la exclusión defensiva, elementos que explicarían una segregación de los MIOs y la presencia de representaciones idealizadas de los otros en situaciones de maltrato en el caso del abuso sexual que, no obstante, no es generalizable a otros tipos de maltrato. Howe (2005) intenta continuar con esta línea de desarrollo a través de la descripción detallada de distintas tipologías y configuraciones de maltrato y sus efectos en los MIOs. Si bien se trata de un gran avance en la relación entre apego y maltrato, la mayoría de sus planteamientos 
TABLA 1

Estudios sobre el efecto del maltrato en las representaciones de apego infantil

\begin{tabular}{llll}
\hline \multicolumn{1}{c}{ Autores } & \multicolumn{1}{c}{ Tipos de maltrato } & Muestra & \multicolumn{1}{c}{ Representaciones apego, instrumento } \\
\hline $\begin{array}{l}\text { Toth, Cicchetti, Macfie \& } \\
\text { Emde (1997) }\end{array}$ & $\begin{array}{l}\text { Negligencia, abuso físi- } \\
\text { co y sexual. }\end{array}$ & $N=107$ & $\begin{array}{l}\text { Representación del self } \\
\text { Representación del otro } \\
\text { MacArthur Story-Stem Battery }\end{array}$ \\
\hline $\begin{array}{l}\text { Toth, Cicchetti, Macfie, } \\
\begin{array}{l}\text { Maughan \& Vanmeenen } \\
\text { (2000) }\end{array}\end{array}$ & $\begin{array}{l}\text { Negligencia, abuso físi- } \\
\text { co y sexual }\end{array}$ & $N=87$ & $\begin{array}{l}\text { Representación del self } \\
\text { Representación del cuidador } \\
\text { Attachment Story Completion Task }\end{array}$ \\
\hline $\begin{array}{l}\text { Waldinger, Toth \& Gerber } \\
\text { (2001) }\end{array}$ & $\begin{array}{l}\text { Negligencia, abuso físi- } \\
\text { co y sexual }\end{array}$ & $N=71$ & $\begin{array}{l}\text { Representación de la relación } \\
\text { MacArthur Story-Stem Battery }\end{array}$ \\
\hline $\begin{array}{l}\text { Venet, Bureau, Gosselin \& } \\
\text { Capuano (2007) }\end{array}$ & Negligencia & $N=74$ & $\begin{array}{l}\text { Calidad de la representación del apego (se- } \\
\text { guro, evitativo, ambivalente, desorganizado). } \\
\text { Attachment Story Completion Task }\end{array}$ \\
\hline
\end{tabular}

Nota: Las muestras de los estudios están compuestas por niños entre 3.8 a 5.8 años de edad. De manera general, los resultados de estos cuatro etudios señalan que existiría un efecto negativo en las representaciones del apego según el tipo de maltrato en comparación con niños que no han sido maltratados.

Fuente: elaboración propia.

no cuentan aun con evidencia empírica contundente que avale sus postulados teóricos.

En base a lo anterior, iqué sabemos a nivel empírico acerca de la relación entre el maltrato infantil y el apego? Aproximadamente el 90\% de los niños expuestos a maltrato desarrollan un apego inseguro, y de este total el $80 \%$ desarrolla un apego desorganizado (Carlson, Cicchetti, Barnett, \& Braunwald, 1989; van IJzendoorn et al., 1999), lo cual se correlaciona con el desarrollo de psicopatologías a corto, mediano y largo plazo (Baer \& Martinez, 2006; Liotti, 1999, 2004; Lyons-Ruth \& Jacobvitz, 1999). Al analizar con detalle los estudios realizados se aprecian dos dificultades fundamentales:

a) La mayoría de estos estudios analizan el apego en términos comportamentales, con metodologías que no permiten apreciar las características cualitativas de los MIOs (Baer \& Martinez, 2006; Hodges \& Steele, 2000; Morton \& Browne, 1998; Toth et al., 2000).

b) El maltrato se define como una variable grupo sin establecer claramente sus tipologías (English et al., 2005), lo que dificulta las comparaciones entre los estudios (Manly, 2005).

Respecto a la evidencia empírica, existen escasos estudios con población infantil que hayan abordado el efecto del maltrato en el apego a nivel representacional (Baer \& Martinez, 2006; Hodges $\&$ Steele, 2000; Morton \& Browne, 1998; Toth et al., 2000; Waldinger, Toth \& Gerber, 2001), demostrado en la siguiente revisión bibliográfica ${ }^{1}$ :

Como se aprecia en la Tabla 1, los tres primeros estudios examinan el apego a nivel representacional e identifican con un sistema de clasificación definido (Maltreatment Classification System de Barnett, Manly \& Cicchetti, 1993) los distintos tipos de maltrato. Si bien se reconoce que existen distintos tipos y niveles de gravedad del maltrato, las muestras no son descritas en esos términos. Las representaciones del apego han sido estudiadas a través del análisis de narrativas que permiten identificar las características de la representación del self, del otro y de la relación de ambos, y los resultados indican que existiría una relación entre maltrato y representaciones de apego, teniendo los

1 La revisión incluyó investigaciones que hubiesen estudiado explícitamente el efecto del maltrato infantil en sus distintas formas, sobre las representaciones del apego en niños evaluadas en términos de representación del self, el otro y la relación, así como la organización del apego. En su metodología los estudios seleccionados cuentan con un grupo de estudio y uno de comparación (por razones metodológicas se excluyen estudios con niños que estén participando de un proceso de adopción). La revisión se realizó en la base de datos ISI Web of Science y en las bases PsycARTICLE, MEDLINE, Psychology and Behavioral Science Collection, y psycINFO a través de EBSCO. Las palabras claves utilizadas en la búsqueda fueron: Child maltreatment, attachment representation, attachment narratives, narratives, representations, child abuse. 
niños maltratados una representación más negativa del self y del otro. Respecto a la evaluación del apego, los instrumentos no permiten describir la calidad del apego en las categorías tradicionalmente utilizadas, lo que dificulta analizar los resultados desde los postulados teóricos antes enunciados. Este último elemento es tomado en cuenta por el estudio de Venet, Bureau, Gosselin y Capuano (2007), quienes clasifican la calidad de las representaciones de apego incorporando los procesos defensivos, no obstante, sólo estudian el efecto de la negligencia, concluyendo que hay una mayor frecuencia de apego inseguro-evitativo en los niños víctimas de negligencia, y que un alto porcentaje de ellos privilegiarían la desconexión del sistema de apego como proceso defensivo. Estos resultados implican un avance en la comprensión de los modelos propuestos y sostienen la hipótesis de que existiría una relación entre los tipos de maltrato, la configuración de los MIOs y los procesos defensivos, no obstante, la relación entre la calidad de las representaciones de apego en términos de los distintos sistemas de memoria y el maltrato no ha sido examinada aun.

\section{Conclusión}

El presente artículo aportó al estudio del maltrato infantil desde la teoría del apego a través de una propuesta crítica que integra a nivel teórico los procesos que forman parte de la configuración del apego en situaciones de maltrato infantil, y los relaciona con los escasos estudios sobre los efectos del maltrato en las representaciones de apego. En este sentido, se señala la necesidad de avanzar en investigaciones que pongan a prueba los modelos teóricos, incluyendo factores que den cuenta de la complejidad del fenómeno del maltrato, haciendo hincapié en la identificación y diferenciación de conductas de maltrato a través de metodologías válidas y confiables. Desde la teoría del apego se requiere implementar metodologías que den cuenta de las distintas manifestaciones y componentes del apego a nivel representacional. Estudios que se orienten en esta línea podrán entregar evidencia sobre la cual sustentar intervenciones orientadas tanto a la detección y prevención del maltrato, como al fomento de contextos para el desarrollo de un apego seguro.
Así mismo, favorece la elaboración de tratamientos psicoterapéuticos especializados y efectivos según el tipo del maltrato, que sean capaces de abordar sus efectos en elementos tales como las defensas, el procesamiento de información, sistemas de memoria, y las estrategias de apego infantil.

\section{Referencias}

Ainsworth, M. D. S., Blehar, M. C., Waters, E. \& Wall, S. (1978). Patterns of attachment: A psychological study of the Strange Situation. Hillsdale, NJ: Erlbaum.

Aspelmeier, J. E., Elliott, A. M. \& Smith, C. H. (2007). Childhood sexual abuse, attachment, and trauma symptoms in college females: The moderating role of attachment. Child Abuse E Neglect, 31, 549-566.

Baer, J. C. \& Martinez, C. D. (2006). Child maltreatment and insecure attachment: a meta-analysis. Journal of Reproductive and Infant Psychology, 24, 187-197.

Barnett, D., Ganiban, J. \& Cicchetti, D. (1999). Maltreatment, negative expressivity, and the development of Type Dattachments from 12-to 24-month of age. Monographs of the Society for Research in Child Development, 64, 97-118.

Barnett, D., Manly, J. T. \& Cicchetti, D. (1993). Defining child maltreatment: The interface between policy and research. In D. Cicchetti \& S. L. Toth (Eds.), Advances in applied developmental psychology: child abuse, child development and social policy (pp. 7-73). Norwood, NJ: Ablex Publishing Corp.

Belsky, J. \& Fearon, P. (2008). Precursors of attachment security. In J. Cassidy \& P. R. Shaver (Eds.), Handbook of attachment: theory, research, and clinical applications (pp. 295-316). New York: Guilford.

Bowlby, J. (1969). Attachment and loss. Vol. 1. Attachment. New York: Basic Books.

Bowlby, J. (1973). Attachment and loss. Vol. 2. Separation: Anxiety and anger. New York: Basic Books.

Bowlby, J. (1980). Attachment and loss. Vol. 3. Loss: Sadness and depression. New York: Basic Books.

Bretherton, I. (1990). Communication patterns, internal working models, and the intergenerational transmission of attachment relationships. Infant Mental Health Journal, 11(3), 237-251. 
Bretherton, I. (2005). In pursuit of the internal working model construct and its relevance to attachment relationships. In K. E. Grossmann, K. Grossmann \& E. Waters (Eds.), Attachment from infancy to adulthood: The major longitudinal studies (pp. 1347). New York: The Guilford Press.

Bretherton, I. \& Munholland, K. A. (2008). Internal working models in attachment relationships: Elaborating a central construct in attachment theory. En J. Cassidy, P. R. Shaver (Eds.), Handbook of attachment: theory, research, and clinical applications (pp. 102-127). New York: Guilford Press.

Carlson, E. A. (1998). A prospective longitudinal study of disorganized/disoriented attachment. Child Development, 69, 1107-1128.

Carlson, V., Cicchetti, D., Barnett, D. \& Braunwald, K. (1989). Disorganized/disoriented attachment relationships in maltreated infants. Developmental Psychology, 25, 525-531.

Cicchetti, D. \& Barnett, D. (1991). Attachment organization in maltreated preschoolers. Development and Psychopathology, 3, 397-411.

Cicchetti, D. \& Toth, S. L. (2005). Child maltreatment. Annual Review of Clinical Psychology, 1, 409-438.

Crittenden, P. M. (1990). Internal representational models of attachment relationships. Infant Mental Health Journal, 11, 259-277.

English, D., Upadhyaya, M. P., Litrownik, A. J., Marshall, J. M., Runyan, D. K., Graham, J. C. \& Dubowitz, H. (2005). Maltreatment's wake: the relationship of maltreatment dimensions to child outcomes. Child Abuse E Neglect, 29, 597-619.

Glaser, D. (2000). Child abuse and neglect and the brain-A review. Journal of Child Psychology and Psychiatry, 41, 97-116.

Hesse, E. \& Main, M. (2000). Disorganized infant, child, and adult attachment: collapse in behavioral and attentional strategies. Journal of the American Psychoanalytic Association, 48, 1097-1127.

Hildyard, K. L. \& Wolfe, D. A. (2002). Child neglect: developmental issues and outcomes. Child Abuse EB Neglect, 26, 679-695.

Hodges, S. \& Steele M. (2000). Effects of abuse on attachment representations: Narrative assessments of abused children. Journal of Child Psychotherapy, 26(3), 433-455.
Howe, D. (2005). Child abuse and neglect: attachment, development and intervention. New York: Palgrave Macmillan.

Jacobvitz, D. \& Hazen, N. (1999). Developmental pathways from infant disorganization to childhood peer relation. In J. Solomon \& C. George (Eds.), Attachment Disorganization (pp. 127-159). New York: The Guilford Press.

Kobak, R. R. (1999). The emotional dynamics of disruptions in attachment relationships: Implications for theory, research and clinical intervention. En J. Cassidy \& P. R. Shaver (Eds.), Handbook of attachment: Theory, research and clinical applications (pp. 21-43). New York: Guilford Press.

Kobak, R. R., Cole, H., Ferenz-Gillies, R., Fleming, W. \& Gamble, W. (1993). Attachment and emotion regulation during mother-teen problem-solving: A control theory analysis. Child Development, 64, 231-245.

Lau, A. S., Leeb, R. T., English, D., Graham, C. J., Briggs, E. C., Brody, K. E. et al., (2005). What's in a name? A comparison of methods for classifying predominant type of maltreatment. Child Abuse ES Neglect, 29, 533-551.

Liotti, G. (1999). Disorganized attachment as a model for the understanding of dissociative psychopathology. In J. Solomon \& C. George (Eds.), Attachment Disorganization (pp. 291-317). New York: Guilford Press.

Liotti, G. (2004). Trauma, Dissociation and Discorganized Attachment: Three Strands of a Single Braid. Psychotherapy: Theory, Research, Practice, Training, 41, 472-486.

Litrownik, A. J., Lau, A., English, D. J., Briggs, E., Newton, R. R., Romney, S. et al., (2005). Measuring the severity of child maltreatment. Child Abuse E3 Neglect, 29, 553-573.

Lyons-Ruth, K., Bronfman, E. \& Atwood, G. (1999). A relational diathesis model of hostile-helpless states of mind: Expressions in mother-infant interaction. In J. Solomon \& C. George (Eds.), Attachment disorganization (pp. 33-70). New York: Guilford Press.

Lyons-Ruth, K. \& Jacobvitz, D. (1999). Attachment disorganization: Unresolved loss, relational violence and lapses in behavioral and attentional strategies. 
In J. Cassidy \& P. Shaver (Eds.), Handbook of attachment (pp. 520-554). New York: Guilford Press.

Lyons-Ruth, K. \& Spielman, E. (2004). Disorganized infant attachment strategies and helpless-fearful profiles of parenting: Integrating attachment research with clinical intervention. Infant Mental Health Journal, 25, 318-335.

Macfie J., Cicchetti D. \& Toth, S. L. (2001). The development of dissociation in maltreated preschoolaged children. Development and Psychopathology, $13,233-54$.

Main, M., Kaplan, N. \& Cassidy, J. (1985). Security in infancy, childhood, and adulthood: A move to the level of representation. In I. Bretherton \& E. Waters (Eds.), Growing points of attachment theory and research. Monographs of the Society for Research in Child Development, 50, 66-104.

Main, M. (1990). Cross-cultural studies of attachment organization: Recent studies, changing methodologies, and the concept of conditional strategies. Human Development, 33, 48-61.

Main, M. \& Hesse, E. (1990). Parents' unresolved traumatic experiences are related to infant disorganized attachment status: Is frightening and/or frightened parental behavior the linking mechanism? In M. T. Greenberg, D. Cicchetti \& E. M. Cummings (Eds.), Attachment in the preschool years (pp. 121-160). Chicago: University of Chicago Press.

Manly, J. T. (2005). Advances in research definitions of child maltreatment. Child Abuse $\mathbb{E}$ Neglect, 29, 425-439.

Mikulincer, M. \& Shaver, P. R. (2008). Adult Attachment and Affect Regulation. In J. Cassidy \& P. R. Shaver (Eds.), Handbook of attachment: Theory, research, and clinical applications (pp. 503-531). New York: Guilford Press.

Moreno Manso, J. M. (2005). Estudio sobre las consecuencias del maltrato infantil en el desarrollo del lenguaje. Anales de Psicología, 22(2), 224-230.

Morton, N. \& Browne, K. D. (1998). Theory and observation of attachment and its relation to child maltreatment: A review. Child Abuse $\mathbb{E}$ Neglect, 22, 1093-1104.

Nelson, K. (1986). Event knowledge: Structure and function in development. Hillsdale, NJ: Lawrence Erlbaum.
Pollak, S. D., Cicchetti, D., Hornung, K. \& Reed, A. (2000). Recognizing emotion in faces: Developmental effects of child abuse and neglect. Developmental Psychology, 36, 679-688.

Shank, R. C. (1982). Dynamic memory: A theory of reminding and learning in computers and people. Cambridge: Cambridge University Press.

Solomon, J. \& George, C. (1999). The place of disorganization in attachment theory. Attachment disorganization. In J. Solomon \& C. George (Eds.), Attachment disorganization (pp. 3-32). New York: Guilford Press.

Toth, S. L., Cicchetti, D., Macfie, J. \& Emde, R. N. (1997). Representation of self and other in the narratives of neglected, physically abused, and sexually abused preschoolers. Development and Psychopathology, 9, 781-796.

Toth, S, L., Cicchetti, D., Macfie, J., Maughan, A. \& Vanmeenen, K. (2000). Narrative representations of caregivers and self in maltreated pre-schoolers. Attachment E Human Development, 2, 271-305.

Tulving, E. (1972). Episodic and semantic memory. In E. Tulving \& W. Donaldson (Eds.), Organization of memory (pp. 381-403). New York: Academic Press.

van IJzendoorn, M. H., Schuengel, C. \& BakermansKranenburg, M. J. (1999). Disorganized attachment in early childhood: Meta-analysis of precursors, concomitants, and sequelae. Development and Psychopathology, 11, 225-249.

Venet, M., Bureau, J., Gosselin, C. \& Capuano, F. (2007). Attachment representations in a sample of neglected preschool-age children. School Psychology International, 28, 264-293.

Waldinger, R. J., Toth, S. L. \& Gerber, A. (2001). Maltreatment and internal representations of relationships: Core relationships themes in the narratives of abused and neglected preschoolers. Social Development, 10, 41-58.

Weinfield, N. S., Sroufe, L. A. \& Egeland, B. (2000). Attachment from infancy to early adulthood in a high-risk sample: continuity, discontinuity, and their correlates. Child Development, 71, 695-702.

West, M. \& George, C. (1999). Abuse and violence in intimate adult relationships: new perspectives from attachment theory. Attachment and Human Development, 1(2), 137-56. 